CARNETS DE Carnets de géographes

GÉOGRAPHES

$7 \mid 2014$

Les espaces de l'entre-deux

\title{
Pour une lecture complexe des espaces portuaires
}

Allan Sekula et Philippe Bazin

\section{Géraldine Millo}

\section{(2) OpenEdition}

Journals

Édition électronique

URL : http://journals.openedition.org/cdg/523

DOI : $10.4000 /$ cdg.523

ISSN : 2107-7266

Éditeur

UMR 245 - CESSMA

Référence électronique

Géraldine Millo, «Pour une lecture complexe des espaces portuaires », Carnets de géographes [En

ligne], 7 | 2014, mis en ligne le 01 juin 2014, consulté le 24 septembre 2020. URL : http://

journals.openedition.org/cdg/523; DOI : https://doi.org/10.4000/cdg.523

\section{(c) $(7)$}

La revue Carnets de géographes est mise à disposition selon les termes de la Licence Creative Commons Attribution - Pas d'Utilisation Commerciale - Pas de Modification 4.0 International. 


\title{
POUR UNE LECTURE COMPLEXE DES ESPACES PORTUAIRES
}

Allan Sekula et Philippe Bazin

\author{
GÉRALDINE MILLO \\ Docteur en Esthétique, \\ Science et technologie des Arts, Université Paris 8 \\ Photographe \\ geraldinemillo@gmail.com
}

\begin{abstract}
Résumé
L'arrivée du container et de l'automatisation dans le transport maritime a conduit à la désaffection des espaces portuaires anciens, devenus terrains vagues quand ils ne peuvent être rattachés à la ville, et à la mise en place de zones entièrement automatisées où les travailleurs se trouvent isolés, solitaires au milieu des containers et des machines. Les espaces portuaires ne sont plus ces lieux de vie marginale et riches d'imaginaires sociaux mais des espaces fermés, condamnant les travailleurs et les citoyens à ne plus rien voir de la matérialité de ce qui circulent sous leurs yeux. Une des conséquences de cette transformation est la perte de l'enracinement matériel, et pour ce qui nous intéresse, spatial : les anciennes zones de débarque et de stockage, et la vie qui allait avec, se trouvent laissées à l'abandon en attendant une hypothétique reconversion, espaces à l'écart où se réfugieront aussi les relégués du capitalisme avancé. Cette transformation des ports et la création d'espaces de l'entre-deux qu'elle engendre, a été abordée de manière différente par deux photographes, Philippe Bazin et Allan Sekula. Chez ces deux photographes, le travail documentaire permet de mettre à jour les causes économiques et politiques qui ont abouties à la formation de ces espaces. Loin de les isoler pour l'étude, ils prennent le parti de tisser un vaste réseau apte à rendre compte de cette nouvelle géographie, tant spatiale qu'humaine. La mise en image documentaire se crée en redondance d'une invisibilité qui travaillent ces espaces : dans les espaces anciens, il n'y a plus rien à voir, dans les nouveaux plus rien n'est donné à voir.
\end{abstract}




\section{Abstract}

The beginning of containers and automation in shipping has led to close down old port areas that became wastelands when they cannot be joined to the city. It also meant completely automated zones where workers found themselves alone among the containers and machines. Port areas aren't these marginal living places where social ideas can be developed anymore. They are closed spaces that condemn workers and citizens to see nothing of the materiality of what passes in front of them. One of the consequences of this transformation is the loss of a material root and, as far as we are concerned, of a special root: the old unloading and storage areas as well as the life that came along with them find themselves abandoned while waiting for a hypothetical reconversion. It therefore is a relegated space where the people banished from advanced capitalism will find refuge. This transformation of the ports and the creation of "in between spaces" to which it leads to has been took up in a different way by two photographs: Philippe Bazin and Allan Sekula. In the documentary work of these two photographs, the economical and political causes that ended up with the formation of these areas are brought to light. They do not only study them but also weave a large network capable of pointing out this new geography, as spatial as human. The documentary picturing is created with the echo of the invisibility that invests these areas: in the older areas there is nothing left to see, and the new ones offer nothing to see. 
Aller à Calais et sentir la ville se vider, les liens urbains se défaire jusqu'à former un espace incompréhensible, des lieux où l'activité gronde au loin, quelque part derrière des kilomètres de grillages, et où des terrains vagues, des zones de stockage plus ou moins vides, des aménagements en déshérence offrent au regard des vides, des creux. À quel imaginaire ce port-là se rattache-t-il ? Celui qui chercherait ici quelque chose de la vie bouillante d'un port international se trouve plongé dans la froideur d'un lieu déserté balayé par le vent. Le mouvement des marchandises échappe au regard, soumis à autorisation, maintenu hors de la vue de celui qui arrive au port à pied. À hauteur de passant, on ne peut voir au loin que des grues semblables à des insectes et d'énormes carcasses de bateau dont seuls les habitués se doutent de ce qu'elles transportent. Et encore. Ni urbains ni portuaires, ces espaces en mal d'affectations nouvelles, en sous-utilisation ou lieux de transit appellent une lecture historique et politique de leur existence. Loin d'être des accidents, lieux non pris en compte ou inatteignables par l'aménagement urbain, ils sont le résultat d'une transformation profonde de la société dont le photographe américain Allan Sekula déploie les ancrages. Ces espaces entre front portuaire et ville doivent être reliés à la question de la transformation du transport maritime imputable à l'arrivée du container, outil d'une véritable rationalisation du transport de marchandise. Un autre photographe, Philippe Bazin, travaille sur ce qui sous-tend ces espaces et qui est un des événements majeurs de notre actualité profonde : le durcissement du capitalisme industriel en capitalisme financier, la mondialisation des échanges qui installent les classes populaires, non dans une solidarité internationale, mais dans la concurrence la plus sauvage. Une lecture du travail de ces deux photographes permettra d'offrir des perspectives pour appréhender des espaces qui se dérobent à la compréhension et à l'expérience.

Allan Sekula, théoricien et photographe, livre deux œuvres majeures, l'ouvrage Fish Story (1995) et le documentaire filmique The Forgotten Space (2010), qui est une manière de film photographique, articulé sur les méthodes de la photographie documentaire.

Philippe Bazin présente plusieurs séries, photographiques et vidéo, sur la thématique des ports, dont la série Porto 2001 qu'il renvoie aux Prisons imaginaires de Piranèse. On peut en effet voir ce travail comme une tentative de déplier un espace dans lequel on ne peut, à première vue, tracer un chemin, une sortie.

Philippe Bazin et Allan Sekula se réclament tous deux d'un réalisme critique, indiquant ainsi une généalogie artistique et sa perspective contemporaine. Les espaces documentaires montrant les espaces maritimes, créés par ces photographes, ne sont pas, comme dans la majorité du genre nommé documentaire, de simples espaces vides propres à recevoir tous les contenus, mais des espaces critiques d'eux-mêmes. C'est cette articulation qui nous mène de la réalité physique des espaces portuaires à sa mise en image, qui nous engage à penser dans deux directions : celle du réel et celle de l'image, espaces arpentés et espaces regardés, pour comprendre l'imbrication des espaces de mise en forme avec les réalités qu'ils montrent. 
Roland Barthes (1980) aura relevé dans La Chambre claire que la force de subversion de la photographie ne réside pas dans ses prouesses formelles mais bien dans son caractère de pensivité, accroché à un détail, un relâchement du cadre. Le montage des images entre elles est alors l'art de connecter entre eux ces espaces de pensivité, ces embrayeurs, pour produire une véritable architecture imaginaire. Ni réelles ni mentales, les photographies sont aussi un espace de l'entre-deux, espaces d'images contenant et le réel et l'imaginé. II y a une affinité élective de la photographie et de ces espaces lacunaires, sur lesquels les deux photographes travaillent: c'est là tout le sens de ce qu'ils nomment réalisme critique, donner à voir ce qui se dérobe, délier l'espace des visibilités, pour produire une expérience, que la philosophe Christiane Vollaire nomme " l'émotion documentaire ${ }^{1}$, apte à offrir une meilleure connaissance de notre actualité. II n'est donc pas étonnant que cette question de la mise en invisibilité revienne dans leurs travaux : les lacunes et les masques constituent le point de départ de leurs recherches, leurs propres embrayeurs de création.

\section{Espaces en creux, ou comment pense la photographie documentaire}

Si la photographie a toujours joué autour du thème de l'absence, elle qui ne fige une présence que pour mieux en révéler le potentiel d'absence, on a vu apparaître depuis une trentaine d'années des travaux portant sur la mise en invisibilité du réel. Dans les années 60 et 70 des photographes tels que Lewis Baltz travaillait sur les correspondances entre mécanique indicielle de la photographie et espaces industrialisés des zones pavillonnaires et des centres commerciaux ${ }^{2}$ pour produire des images minimalistes aux formes simples et géométrisées, la génération de la fin des années 70 , et du milieu des années 80 , avec l'apparition des nouvelles technologies, fait correspondre langage photographique et perte de l'expérience concrète du monde. La prévalence n'est plus donnée au caractère mécanique du medium mais à son statut d'indice, à ses capacités dynamiques de relevé fragmentaire. On ne peut comprendre l'importance de cette photographie et sa différence avec les formes standardisées du documentaire courant ou avec le reportage, si l'on n'entend pas que son objet est la formation d'espaces de pensée et nom la délivrance de contenus narratifs. Alors que Sekula ne cesse d'articuler images et textes pour créer de complexes structures documentaires ${ }^{3}$, Bazin minimalise l'image en radicalisant l'esthétique documentaire. Dans les deux cas, les questions de ce qui est donné à voir, de ce qu'ils donnent à voir et de comment ils le présentent ont un ancrage éminemment politique. Critique est menée des limites de notre organisation sociale en même temps que des limites de l'image. C'est grâce à une telle approche qu'une autre lecture peut être faite des espaces portuaires à l'affectation incertaine. Elle engage en effet le lecteur dans un parcours des espaces symboliques et matériels qui façonnent de tels lieux.

\footnotetext{
${ }^{1}$ Entretien avec Christiane Vollaire, in MILLO (2013).

${ }^{2}$ Voir le site, http://www.americansuburbx.com/channels/l/lewis-baltz, qui présente un certain nombre de ses œuvres.

${ }^{3}$ Voir le site de sa galerie française, http://michelrein.com/fr/artistes/portfolio/1/Allan\%20Sekula
} 
La radicalisation de la forme documentaire, frontalité de la prise de vue, netteté et éclairage neutre, permettent à Philippe Bazin ${ }^{4} d^{\prime}$ insister sur ce que nous ne pouvons plus voir : que le réel, son appropriation collective et individuelle, nous sont confisqués; que par des mécanismes plus structurels que véritablement intentionnels, nous nous sommes retirés des espaces urbains, que nous ne voyons plus, parce que nous ne l'expérimentons plus, la lourdeur de la matière et la pesanteur de l'espace concret, que des espaces lacunaires que nous pourrions nous approprier sont considérés à tort ou à raison comme interdits. L'espace public n'est alors plus que celui que nous traversons, ou celui où nous nous divertissons dans des zones aménagées pour cela. Des vides, des creux, nous ne savons pas que faire : mieux, ils nous font peur car ce sont les seuls espaces que les autres invisibles, les émigrants clandestins, trouvent où vivre, survivre. Peur du vide et peur de l'autre, antiques creuset des peurs humaines, appartiennent structurellement à notre organisation politique. Une fréquentation de l'ensemble de l'œuvre de Bazin, offre une réflexion poussée sur le sens, la symbolique de ces espaces incertains. Dans la mesure où ceux-ci sont une imbrication de réalités physiques et de réalités mentales, il est nécessaire que de tels travaux documentaires les mettent en écho.

Les réalités des espaces portuaires de l'entre-deux ne sont pas nécessairement abordées frontalement, mais apparaissent en creux dans les différentes séries. Si la série Dans Paris semble bien loin de ce sujet précis, il n'est qu'à rappeler qu'elle traite en creux de notre rapport à l'espace public urbain et en face de l'invisibilité de clandestins qui suivent les mêmes routes que le transport de marchandise. Les espaces de l'entre-deux, ces espaces invisibles où ils sont maintenus et que nous ne voyons pas alors que nous les traversons tous les jours, constituent le même continuum que les espaces incertains des zones portuaires. Là, comme ailleurs, les creux permettent d'accueillir, ou plutôt de réceptionner, de stationner les flux migratoires, comme mercantiles. Ainsi le dialogue des séries entre elles relie ces espaces portuaires de l'entre-deux à la ville et à la mer, à la politique et à l'économie, à l'organisation et au flux... Nous proposons donc un montage en ellipse Photographie 1 et Photographie 2 de deux séries, Dans Paris (2009-2011) et Porto (2001), et invitons à se reporter aux travaux de Philippe Bazin sur les flux au sein de différents ports et à ceux portant sur les politiques migratoires.

\footnotetext{
${ }^{4}$ Voir le site de l'artiste, http://www.philippebazin.fr.
} 
Photographie 1 : Philippe Bazin, Dans Paris, 2009-2011

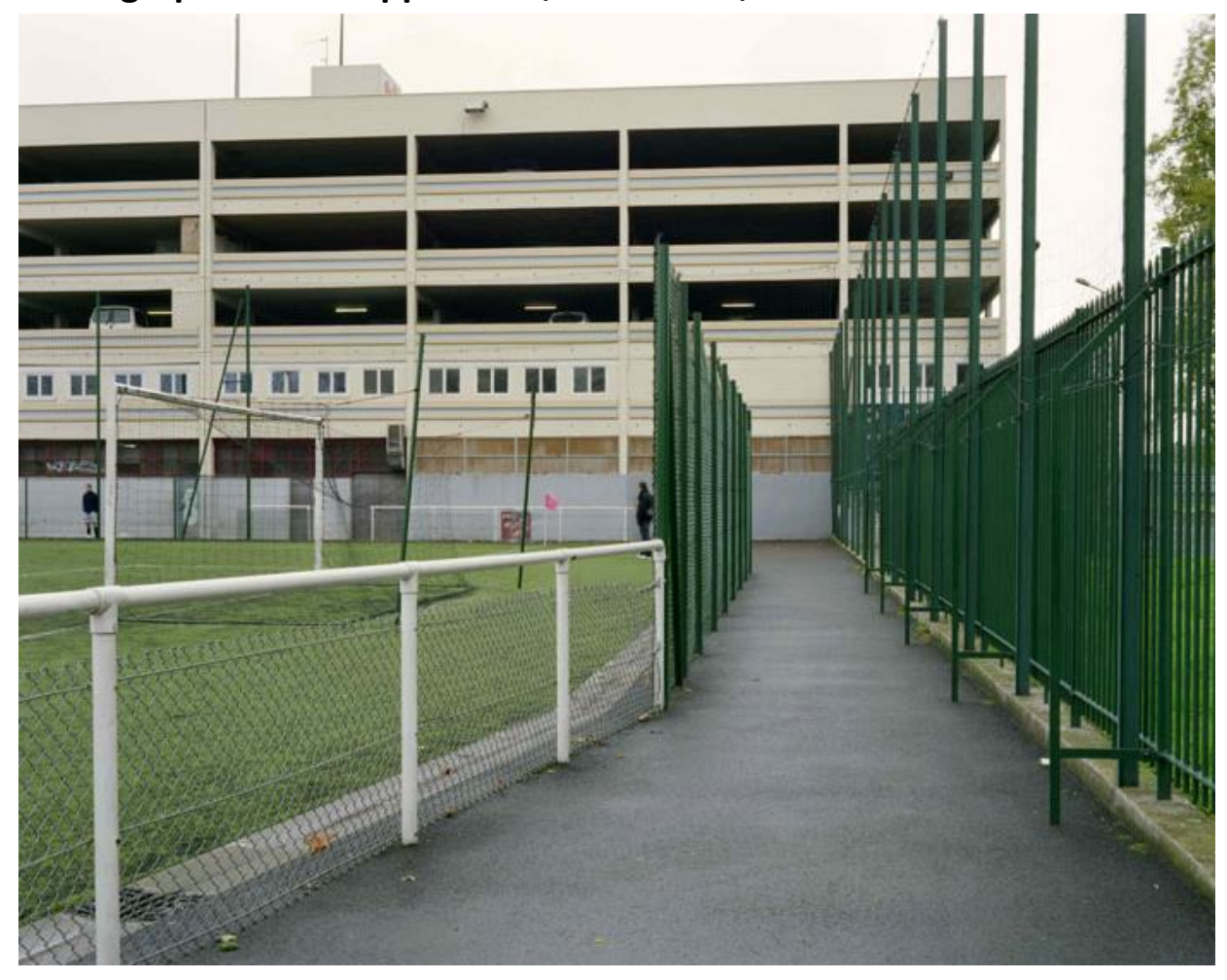

Photographie 2 : Philippe Bazin, Porto, 2001

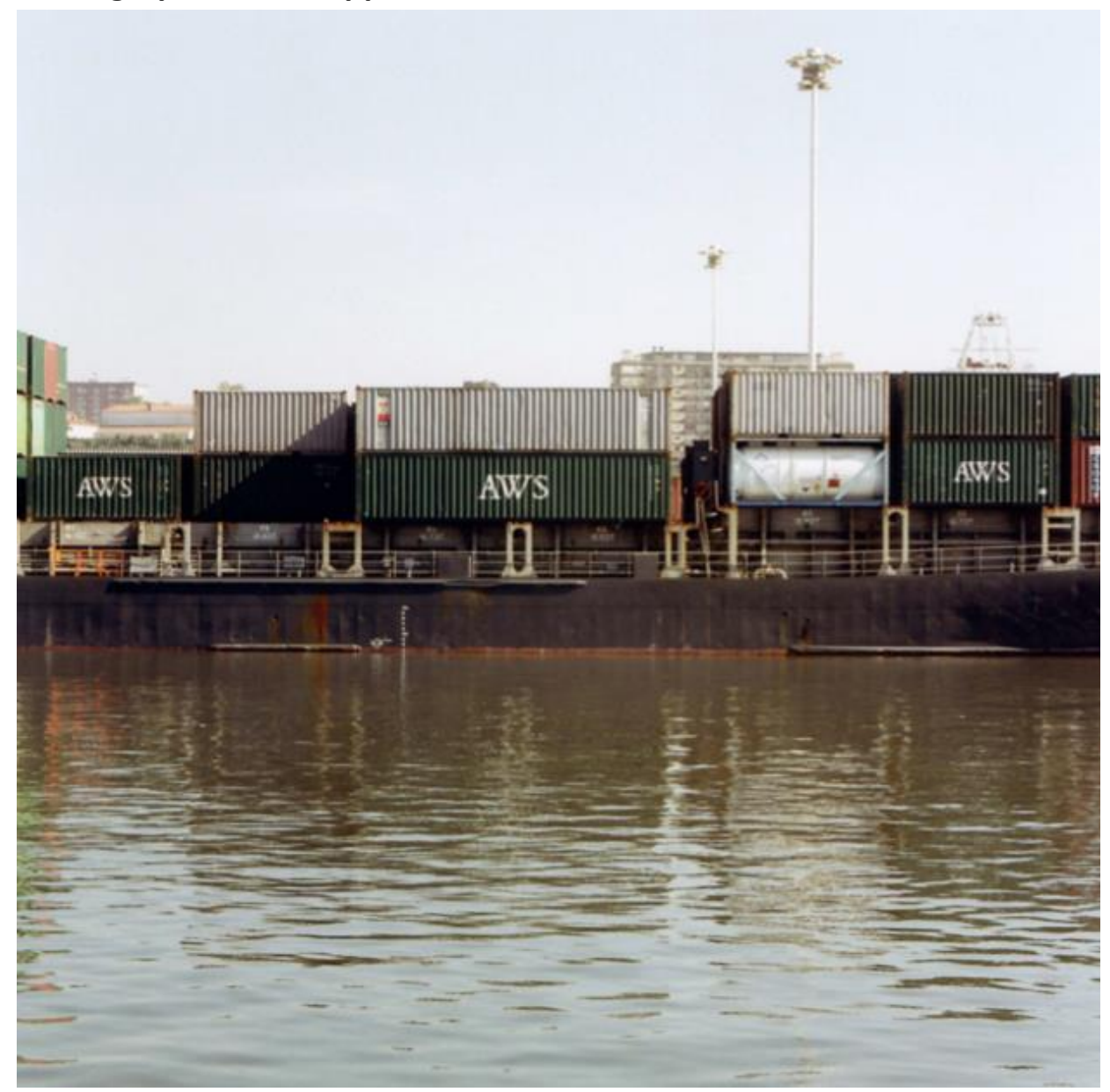


Dans ces deux images, l'entre-deux n'est pas une géographie visible, mais se dessine au contraire en filigrane. Photographie 1 , il se dessine dans l'espace vide créé par un passage entre un stade et un espace vert, espace entre deux barrières où les afghans se retrouvent, espace d'un réseau qui transforme, ainsi que le note Bazin, ces espaces lâches, en expérience autant poétique que politique ${ }^{5}$. Photographie 2 , le front portuaire joue comme une barrière isolant la ville de la mer, masquant, protégeant, isolant hermétiquement la cité des réalités économiques. On devine alors les espaces entre les deux, entre le port et la ville, comme des espaces tampons, des espaces où se manifestent la difficulté de raccorder l'usine des flux à la cité, manifeste et symbole des tensions entre économie et politique. Ce sera aussi dans ces espaces-là, derrière le port, en marge des villes, que l'on retrouvera ceux qui sont absents des images, les femmes et hommes de l'entre-deux ${ }^{6}$. Dans Porto 2001, Bazin monte des images d'ouvriers, des images à l'intérieur du port et des images de ses abords. Rapporté à la question des espaces maritimes, photographier le port qui s'offre comme une muraille avant la ville quand on arrive de la mer, et des espaces désolés ou surchargés de travail mécanique, c'est dessiner une géographie mentale des ports contemporains, où s'alternent et s'imbriquent la violence d'une barrière qui cache au regard le labeur toujours plus épais de ceux qui y travaillent, et l'impossibilité d'habiter, c'est-à-dire de se mêler et de s'approprier physiquement et symboliquement ce monde-là.

Cette distorsion entre la réalité concrète du monde et l'image que nous en avons est aussi ce sur quoi travaille Allan Sekula, par d'autres moyens. II n'use pas de la radicalisation de la forme stricte du document mais au contraire convoque une esthétique de l'instantané et un montage d'images où se télescopent des réalités en apparence hétéroclites. Ainsi la rationalisation du transport maritime et, en conséquence, des espaces portuaires se trouve relayée dans un complexe montage d'images convoquant des réalités géographiquement éparses et des usages multiples des espaces, selon que ceux-ci appartiennent au nouveau ou à l'ancien régime maritime. N'offrant aucune compréhension immédiate, l'ensemble des images invitent à prendre le temps du parcours, à retourner, non pas à un spectacle des images, mais à une lecture : c'est depuis cet espace en creux que les images pensent et nous font réfléchir.

\footnotetext{
${ }^{5}$ Philippe Bazin: "Comme le souligne Denis Lemasson, les clandestins (Afgans ou autres) ont réinvesti poétiquement les rues de Paris, rues désertées de cet habiter poétique par les parisiens eux-mêmes, à l'heure où tous les espaces publics sont réaménagés en vue du tourisme et de l'activité commerciale. L'invisible se situe donc là, non pas seulement un clandestin qu'on ne voit pas alors que toutes les conditions politiques organisent cette invisibilité, mais aussi une poétique qui maintenant nous échappe.", in http://www.philippebazin.fr/index.php?/travaux-recents/un-camp-afghan-paris/.

${ }^{6}$ On pourra se reporter ici au livre de Philippe Bazin et de la philosophe Christiane Vollaire, Le Milieu de nulle part (2012), qui porte sur des centres de réfugiés tchétchènes en Pologne. La philosophe, pour les entretiens qu'elle a menés, est partie de trois questions qui indiquent clairement cet entre-deux : d'une part, d'où venezvous, d'autre part où allez-vous, et enfin cette dernière qui ouvre véritablement sur un possible habiter, une appropriation d'espace : que souhaitez-vous.
} 
Rationalisation du transport maritime et formation d'espaces informes : documenter le néocapitalisme

La formation d'espaces de l'entre-deux n'est pas le propre du néocapitalisme. Si ces espaces se sont multipliés et surtout ont pris sens avec la montée d'une urbanisation appuyée sur l'industrialisation, les espaces auxquels s'est intéressé Allan Sekula sont proprement le résultat d'une transformation imputable au néocapitalisme boursier. En effet, la création d'espaces adaptés à la rationalisation des modes de production a abouti à la création d'espaces périphériques délaissés, zones oubliées des aménagements urbains, entre voies de circulation, villes et centres de production. L'arrivée du container a permis au port d'entrer dans cette danse de la maitrise de l'espace et de la matière et a abouti à transformer la géographie des lieux de manière significative. De la sorte, nombres de terrains autrefois rattachés à la ville de manière organique, se sont retrouvés sans utilité, simples espaces de transfert, depuis la mer jusqu'aux axes routiers et ferroviaires. Que ces espaces de l'entre-deux soient devenus des non-lieux est dû à un double facteur : d'une part l'inadaptation de certains lieux, d'autre part la disparition de la présence concrète et voyante des marchandises. Ainsi si certains terrains se trouvent encore parcourus par des camions ou des wagons de fret, ce sont des espaces qui ne transpirent plus ce qui les traversent. S'y promener ne permet pas de prendre la mesure des matières qui y passent, mais n'offre qu'un vide auquel répond en écho l'opacité des containers.

La mondialisation des échanges délocalise l'espace concret de telle sorte que celui-ci apparaît toujours en fuite, toujours repoussé dans un infini potentiel. Le propre du néocapitalisme est d'avoir poussé à la limite la primauté au capital et de réduire les échanges de marchandise aux échanges boursiers. Ainsi la dimension abstraite de la bourse contamine les espaces concrets, de telle sorte qu'il n'y a plus rien à voir dans les ports. Allan Sekula met en relation la forme du container et la transformation du port : le container ressemble au billet de banque - dimension abstraite de l'échange - et le port n'a plus d'odeur :

"Avant, les illusions des sens poussaient les habitants des ports à croire qu'une économie mondiale pouvait se voir, s'entendre et se humer. La richesse des nations défilaient dans la passe (...). Mais plus le mouvement des biens dans les ports est régularisé, véritablement conteneurisé, autrement dit plus il est rationalisé et automatisé, et plus le port ressemble à la bourse. Un aspect phénoménologique capital est ici l'élimination des odeurs. Les marchandises qui puaient autrefois (...) circulent à présent dans des boites. Les boîtes vues d'en haut ont les proportions de billets de banque légèrement allongés. Le contenu anonyme - composants électroniques, bien monnayables des demandeurs d'armes, cocaïne, vieux papiers (qui peut le savoir?) - se cache derrière les parois en tôle d'acier ondulée marquées aux logos des compagnies de navigation internationales, Evergreen, Matson, American President, Mitsui, Hanjin, Hyundai.» (Sekula, 1995 : 4) 
L'espace alors se transforme et la vie des espaces portuaires dont nous gardons l'imaginaire au travers des livres n'a plus que peu ou prou de rapport avec la réalité. Sekula relève que la mer est donnée en spectacle, les docks réaménagés en appartement avec vue sur la mer ou en zones commerciales de promenade, tandis que le port comme espace de travail est relégué loin de la ville et vidé de ses travailleurs. Si la conséquence pour les ouvriers est l'isolement de ceux qui sont en poste et le chômage pour les autres ${ }^{7}$, la conséquence est aussi la perte de contrôle par les populations de ce qui circulent et du rapport concret au monde. Les travailleurs des ports eux-mêmes ne savent plus ce qu'ils transportent, et à quelles fins. L'aliénation qui réside dans la perte des fins de la production et le cantonnement dans la force de travail (physique de l'ouvrier ou intellectuelle de l'employé) trouve un puissant allié dans cette perte de confrontation avec la matérialité concrète et aliène aussi ceux qui n'y sont plus confrontés.

Georges Bataille avait eu dans la revue Document cette formule à propos des abattoirs, lieu archétypal de la confrontation de la matière et du rationnel :

" de nos jours l'abattoir est maudit et mis en quarantaine comme un bateau portant le choléra. Or les victimes de cette malédiction ne sont pas les bouchers ou les animaux, mais les braves gens eux-mêmes qui en sont arrivés à ne pouvoir supporter que leur propre laideur, laideur répondant à un besoin maladif de propreté, de petitesse bilieuse et d'ennui " (Bataille 1929).

Le matérialisme politique de Marx, auquel Sekula renvoie, prend pour postulat philosophique l'enracinement des structures symboliques dans l'organisation matérielle de la production. Bataille tire les conséquences de cette approche en montrant comment la tentative d'éradication de la basse matérialité entraine l'aliénation des consciences. Rendre invisible la part laborieuse en la maitrisant dans des processus d'automatisation et de conteneurisation : tel est le projet de l'usine, dont Sekula dira que, dans ses capacités de délocalisation, elle ressemble davantage au bateau, et le bateau, dans sa lourdeur et ses liens robotisés au port, à l'usine. La lecture éclairée des espaces portuaires concrets, que le photographe permet par ses montages, donne à saisir ce qui n'est pas immédiatement visible dans ces espaces entre nouveaux ports automatisés et ville portuaire, et de même que " l'ancienne façade de port, que le chômage a coupé de ses liens avec un fond culturel commun, passe au service d'une rêverie bourgeoise sur le passé mercantile " (Sekula, 1995 : 12), l'espace désormais séparé des zones automatisés et de la ville exprime l'actualité du néocapitalisme.

Fish Story est le troisième volet d'un "cycle consacré aux géographies imaginaires et matérielles du capitalisme avancé " (Sekula 1995: 202). Le processus d'enquête dans la photographie documentaire peut être rapproché de celle mise en œuvre dans les sciences sociales, mais l'inscription du travail dans le champ de l'art indique une prise de liberté et de distance par rapport aux méthodes scientifiques. II ne s'agit pas pour le photographe de parcourir méthodiquement les différents champs visuels qui lui semblent être des indices de l'organisation maritime dans le capitalisme avancé, mais de produire une méthode mixte :

\footnotetext{
${ }^{7}$ Ce qui sera le cœur de son documentaire filmique, The forgotten space, DVD Wild Art Film, 2010.
} 
"Les lieux étaient choisis sur des coups de tête, aussi bien que pour des raisons thématiques. En général, ces choix s'appuyaient sur la recherche de pôles présents et passés de la puissance maritime, et de territoires plus périphériques, souvent soumis à un pouvoir unique, tel le Mexique, ou pris entre des conflits entre plus puissants qu'eux, par exemple la Corée et la Pologne » (Sekula, 1995 : 202)

L'organisation de l'enquête, sa planification, repose donc à la fois sur une appréhension rationnelle de la question, à la fois sur ce que Sekula appelle des coups de tête et qui jouent le rôle de court-circuitage d'une géographie abordée de manière systématique. Sekula transcende les frontières et inscrit son travail à la fois dans une analyse théorique d'inspiration marxiste, à la fois dans une impulsion subjective dont les traces émaillent l'ensemble du parcours. Il ouvre d'ailleurs la partie textuelle par cette déclaration : "Grandir dans un port, cela prédispose à nourrir des idées bizarres sur la matière et la pensée. Là, je ne parle que pour moi, encore que j'imagine qu'une certaine insistance pessimiste et obstinée sur la primauté des facteurs matériels soit ancrée dans la mentalité de tous les habitants des ports. " (Sekula, 1995: 12). Le réalisme photographique des images qui accompagnent ces textes est tout aussi complexe, dans la mesure il parcourt moins un ensemble visuellement cohérent, que des lignes de force qui relient des réalités éparses entre elles.

Qu'ont en effet à voir ensemble dans une même série les vestiges d'un plateau de tournage sur un chantier naval désaffecté et un quartier coréen à Los Angeles ? Le réalisme de ces images articule de manière critique ce que le texte analyse :

"L'espace se transforme. Les profondeurs marines sont sonorisées. Des bateaux de pêche disparaissent dans la mer d'Irlande, précipités au fond par des sous-marins. Les hommes d'affaires dans leurs avions lisent des romans passionnants où il est question de sonars. On démolit les bordels sur les quais ou on les rénove pour en faire des appartements. On convertit des chantiers navals en plateaux de tournage » (Sekula, $1995: 12$ ).

Ce n'est donc pas un ensemble dont la cohérence est à rechercher dans une continuité visible : le propre du réel est d'être éclaté au quatre coins du globe et la réalité des ports d'être masquée par sa mise en boite et sa mise à l'écart des villes. Ainsi l'illusion qu'avaient les habitants des ports de pouvoir voir l'économie mondiale défiler sous leurs yeux se trouvent-elles remplacées par un contenu anonyme caché dans les conteneurs. De même, les bateaux arrivant ne sont plus les reflets de puissances économiques et politiques, mais des groupements de pays, dont l'un possède le pavillon, l'autre la marchandise, encore un autre l'équipage et enfin un dernier le capitaine. Les images confrontent ainsi ces réalités visibles entre elles: la manière dont elles n'ont rien à faire ensemble mais se trouvent indéfectiblement liées révèlent les rapports de forces qui se jouent dans le façonnage de la réalité visible que sont les ports et ce qui les sous-tend, la structuration économique du capitalisme avancé. Les espaces de l'entre-deux qui émaillent les grands ports 
contemporains ne peuvent acquérir une lecture pertinente que dans la mesure où ces lieux sont rattachés aux tensions qui les ont créées. Aussi, l'envergure et la richesse du travail de Sekula est justement d'éclairer une problématique, le transport maritime, peu médiatisée, mais qui façonne nos espaces de vie : une géographie des flux confrontés aux réalités d'ici et maintenant des espaces terrestres et humains.

Photographie 3: Allan Sekula, « Un chantier naval désaffecté utilisé par le corps expéditionnaire des Marines pour l'entraînement "contre-terroriste ". Port de Los Angeles. Wimmington, octobre 1992. ", Fish Story, 1995.

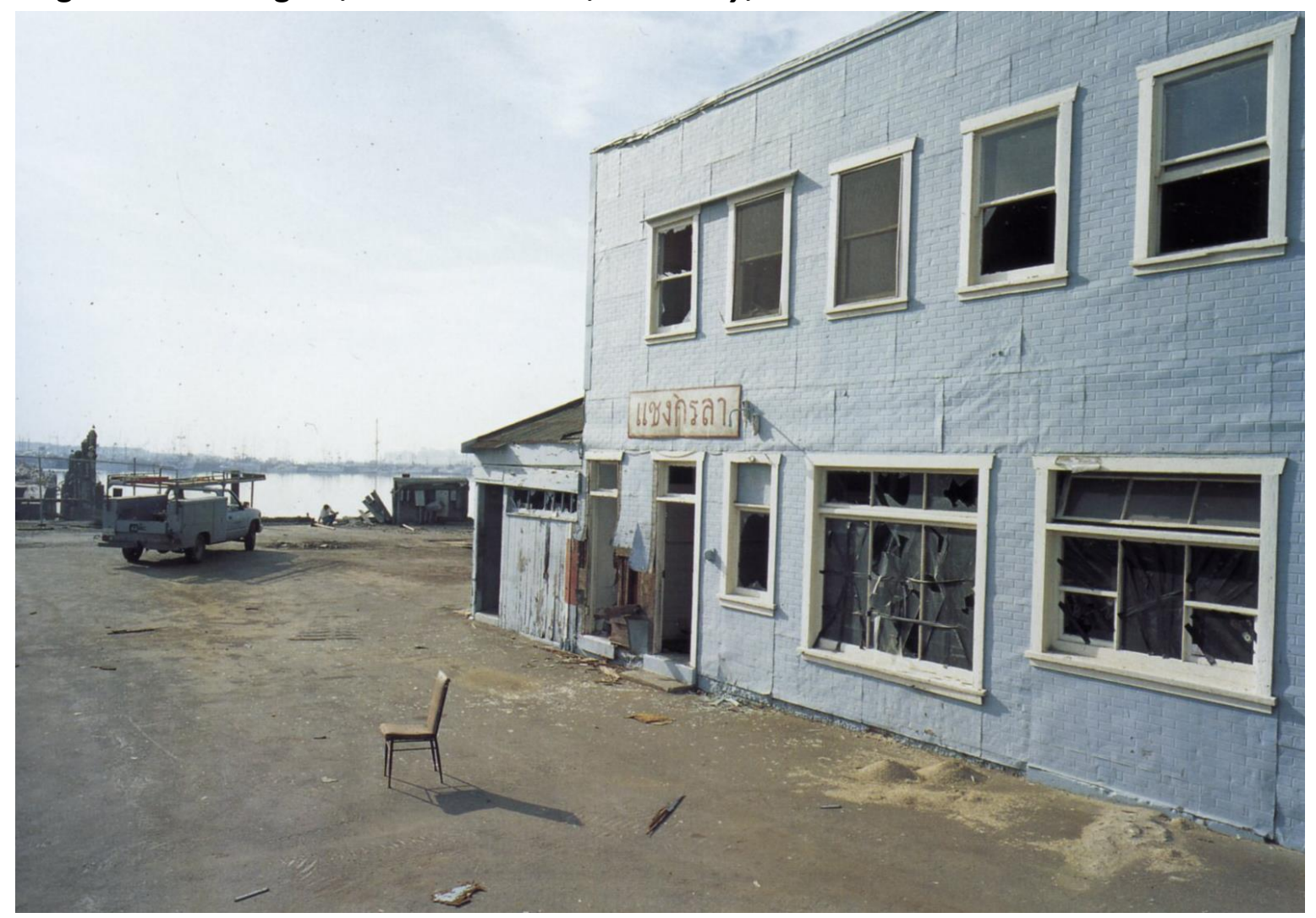


Photographie 4: Allan Sekula, "Essai d'un camion-robot conçu pour déplacer les conteneurs à l'intérieur de la gare maritime ECT/Sea-Land entièrement automatisée. Maasvlakte. Port de Rotterdam, septembre 1992. ", Fish Story, 1995.

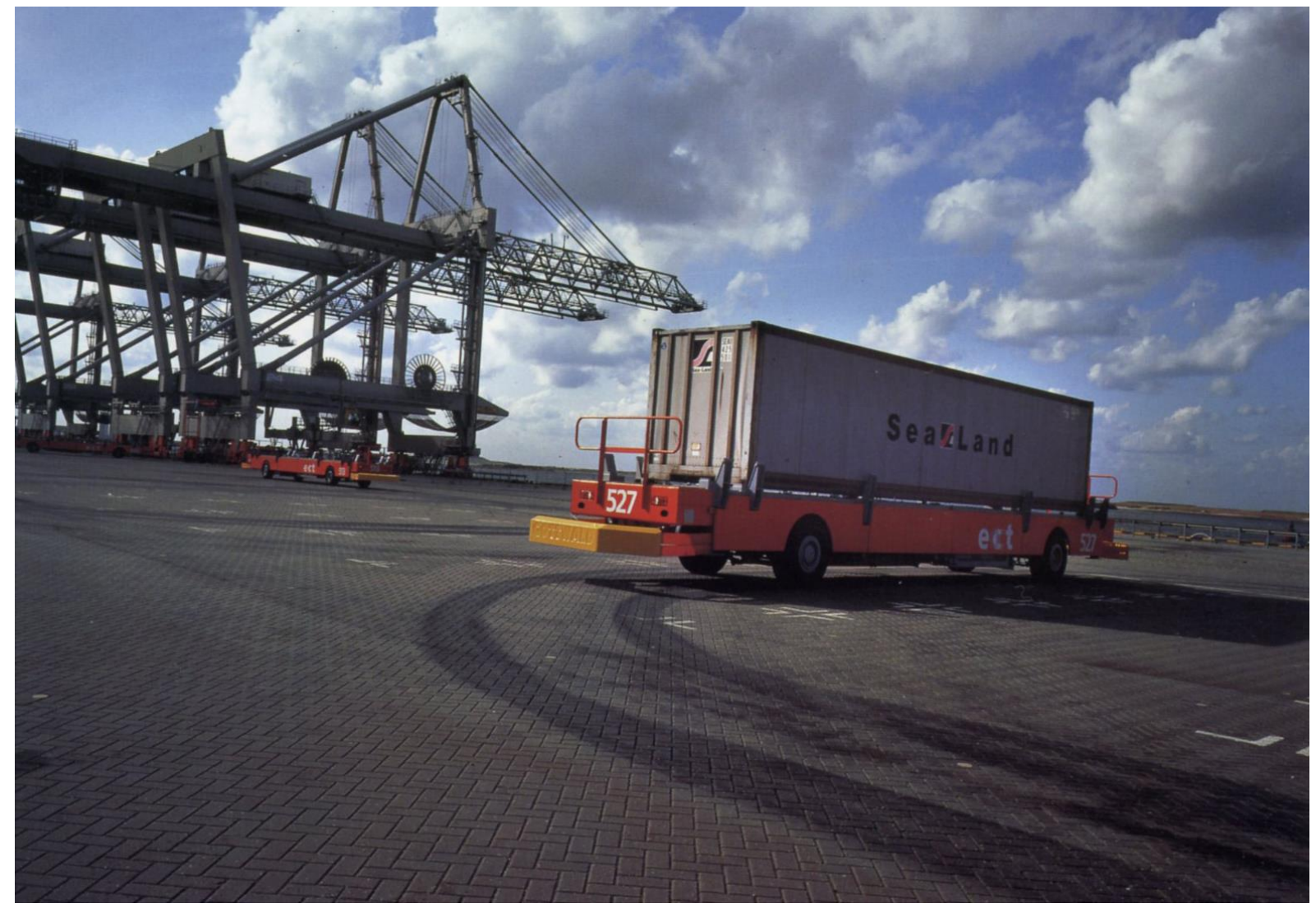

Photographie 5 : Allan Sekula, « Panorama. En mer. », Fish Story, 1995.

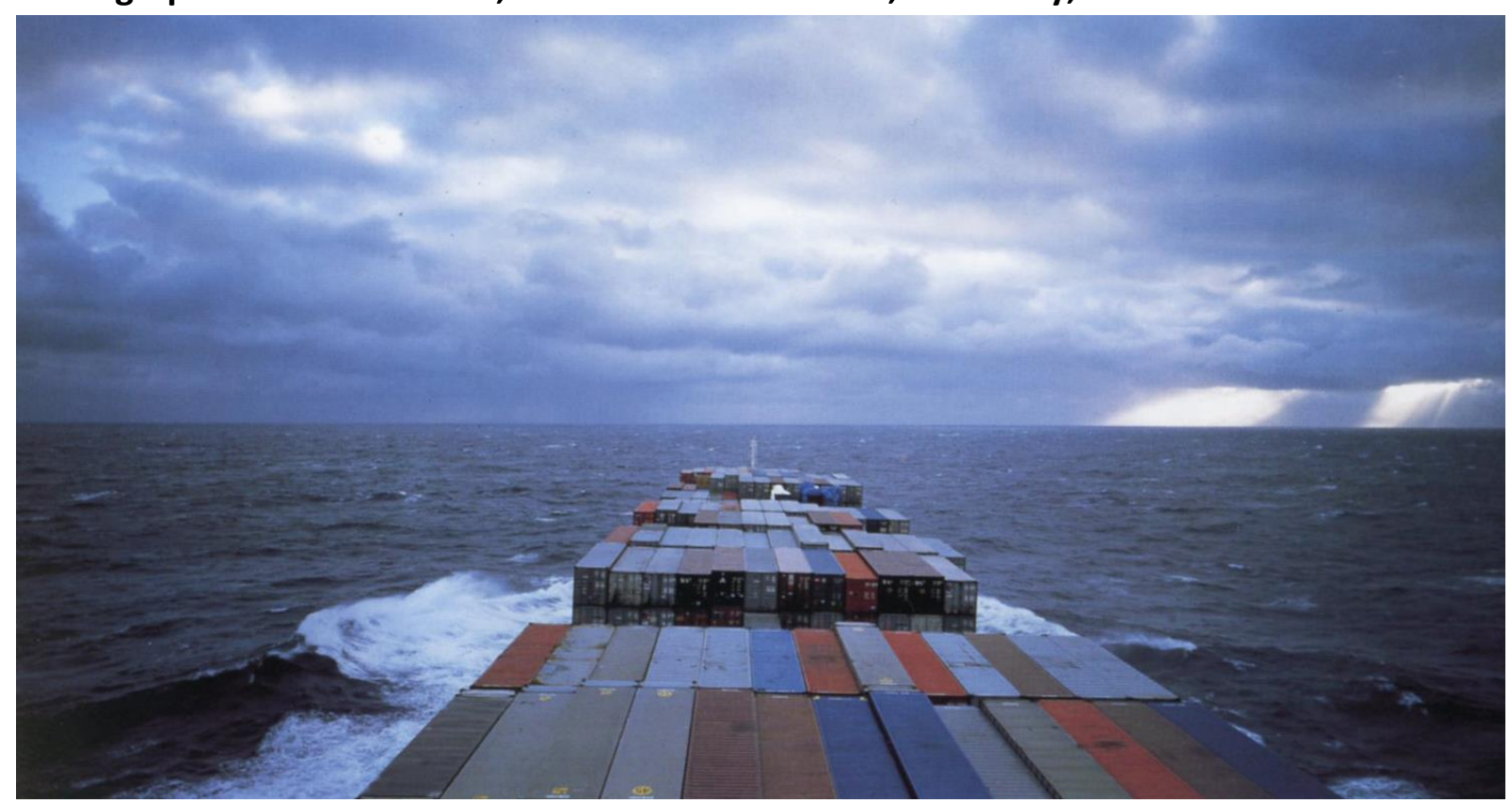


Par la fragmentation et le montage, ou la frontalité et la séquence, Allan Sekula et Philippe Bazin nous permettent d'apprendre à lire la raison d'être de ces espaces et le théâtre qu'ils masquent-révèlent. Sekula les inclue dans une enquête générale sur le transport maritime tel qu'il est relié à l'organisation économique de la production et des échanges commerciaux du néocapitalisme. Philippe Bazin a une approche plus foucaldienne de cette question, en s'intéressant et en mettant en lien différents champ d'action d'une même force : ainsi son travail explore-t-il la forme des ports comme la question des politiques migratoires, la forme du travail comme le rapport entre institutions et singularités individuelles. Ces modes de travail documentaire créent des espaces de pensée plus que des récits narratifs dans lequel la photographie documentaire est abordée comme un espace-carrefour. Ces espaces de l'entre-deux qui jalonnent les ports sont les conséquences et symptômes d'une histoire que leurs images étirent et déroulent.

\section{Bibliographie}

BARTHES R. (1980), La chambre claire, Note sur la photographie, Paris, éd. De l'Étoile, Gallimard, Le Seuil.

BAZIN P. (2006), Dufftown, Rouen, POC.

BAZIN P., VOLLAIRE C., (2012), Le Milieu de nulle part, Grânes, Créaphis éditions.

MILLO G., (2013), thèse de doctorat, Pour une poétique du document. Esthétique et politique de la photographie, Paris 8.

SEKULA A., (1995), Fish Story, Düsseldorf, Richter Verlag.

SEKULA A., (2005), in Document 3, Le statut de l'auteur dans l'image documentaire: signature du neutre, Paris, Jeu de Paume.

SEKULA A., (2010), The forgotten space, DVD Wild Art Film.

Documents, réédition intégrale de la revue, deux tomes, Paris, éd. Jean-Michel Place, 1991.

Revue Communication, (2001), $n^{\circ} 71$, Paris, Seuil.

\section{Sites internet}

http://www.philippebazin.fr

http://www.americansuburbx.com/channels///lewis-baltz

http://michelrein.com/fr/artistes/portfolio/1/Allan\%20Sekula 